\title{
Bridge Construction Progress Monitoring Using Image Analysis
}

\author{
Changyoon $\mathrm{Kim}^{1}$, Hyoungkwan $\mathrm{Kim}^{2}$ and Yeonjong $\mathrm{Ju}^{3}$ \\ 1School of Civil and Environmental Engineering, College of Engineering, Yonsei University, 134 Shinchon- \\ dong, Seodaemun-gu, Seoul 120-749, Republic of Korea; PH (+822) 2123-7940 ; FAX (+822) 364-5300; e- \\ mail: changyoonkim@yonsei.ac.kr \\ ${ }^{2}$ Associate Professor, School of Civil and Environmental Engineering, College of Engineering, Yonsei \\ University, 134 Shinchon-dong, Seodaemun-gu, Seoul 120-749, Republic of Korea; PH (+822) 2123-5799; \\ FAX (+822) 364-5300; e-mail: hyoungkwan@yonsei.ac.kr \\ ${ }^{3}$ School of Civil and Environmental Engineering, College of Engineering, Yonsei University, 134 Shinchon- \\ dong, Seodaemun-gu, Seoul 120-749, Republic of Korea; PH (+822) 2123-7940 ; FAX (+822) 364-5300; e- \\ mail: j970132@hotmail.com
}

\begin{abstract}
Construction progress monitoring is a critical task for construction managers. Traditionally, site superintendents walk the construction site to verify the progress report and understand the current state of construction processes. To get rid of the time consuming process, this research proposes a construction progress monitoring system comprised of a network type closed-circuit television (CCTV) camera, a wireless local area network, and image processing techniques. A civil engineering construction site was selected and the system is able to capture the images of the construction processes. In this paper, the hardware architecture of the system and its initial application results are presented with some promising outcomes.
\end{abstract}

\section{Introduction}

Construction progress monitoring is a critical task for construction managers. Successful progress monitoring prevents construction projects from falling into the pitfalls of ineffective management. Nowadays, many construction projects are provided with various photogrammetry equipments such as digital camera and closed-circuit television (CCTV) camera to record the pictures of construction sites. These saved images are typically used only for documentation of daily or weekly construction processes. Coupled with image processing techniques, the images can potentially be used to produce information of certain activity progress. In this paper, a research effort is introduced where a CCTV camera-based system is used to monitor the construction operations of a cable stayed bridge. The image processing system is developed in conjunction with the 3D (Three-Dimensional) CAD (Computer-Aided Design) model, in order to accurately and automatically track the construction progress level of the segment between two main pylons. To verify the construction progress monitoring methodology, tests are conducted at the cable-stayed bridge construction site.

\section{Research Background}

Image processing technique is gaining increased recognition in construction material engineering areas. Kim et al. (2002; 2003; 2004), with the use of laser technology, captured 3D image data of construction aggregates, and analyzed the images with advanced imaging algorithms to characterize the quality of the construction materials. Abdel-Qader et al. (2003) compared diverse image processing techniques chose a series of techniques that work best for crack identification in a bridge. By combination of image processing techniques and a database of construction materials, Brilakis and Soibelman (2008) used a shape retrieval mechanism to recognize a range of construction material resources.

On the other hand, image process techniques have been used for analyzing construction project processes. Zou and Kim (2007) suggested a strategy for analyzing idle time of hydraulic excavators, using the hue, saturation, and value color space. Quiñones-Rozo et al. (2008) applied image processing techniques for identifying and quantifying the excavating area. Bosche and Haas (2008) obtained promising results of 
progress monitoring using a 3D laser scanner and a simple 3D model. These efforts have shown a big potential for imaging techniques to produce the essential construction management information in an effective manner.

\section{System Architecture for Wireless Image Data Acquisition}

The images of the bridge construction processes are automatically transferred to the head office. Fig. 1 shows the system architecture for image data acquisition mechanism. A $704 \times 480$ network CCTV camera was installed at the cross beam of the pylon. This network CCTV camera can directly be connected to the internet, so that the acquired data are easily transferred to end users. However, due to the harsh construction environment, it is not easy to transfer the images through a direct LAN (Local Area Network) cable. It is possible that some heavy construction equipment or other construction vehicles damage the LAN cable.

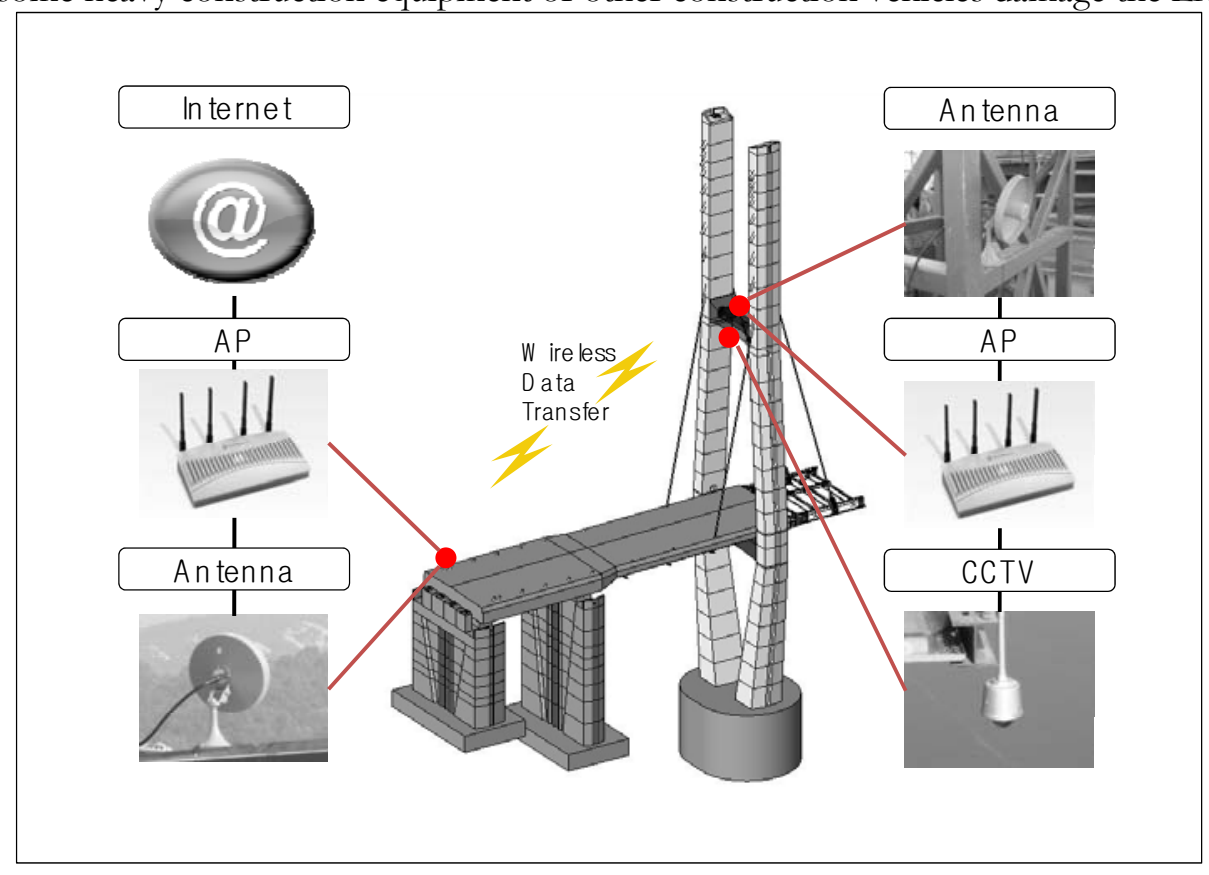

Fig. 1. Automated wireless image data acquisition system

Two access points (devices that allow for wireless communication) are used: one for data transmission and the other for data reception. One access point was installed to be connected to the camera at the cross beam of the pylon and the other to be connected to the internet at the entrance of the construction site (the end of the side span), resulting in the establishment of the Wireless LAN (WLAN) environment. To cover the communication distance of about $68.4 \mathrm{~m}$ between two access points, two directional antennas were used. The image data which acquired by the CCTV camera was successfully transferred through the WLAN system. Construction managers at the head office in a remote area can monitor the construction progress of the cable stayed bridge in a real time via the internet connection. For documentation purpose, the images are being stored at the main storage device in Seoul, in every two seconds.

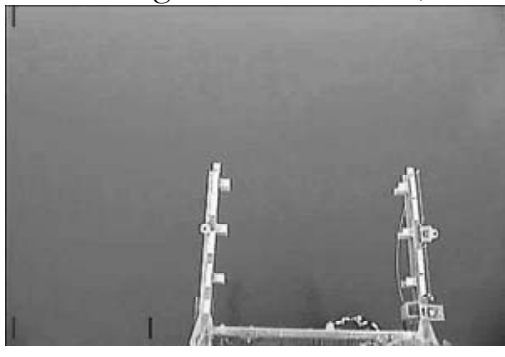

(a) Day

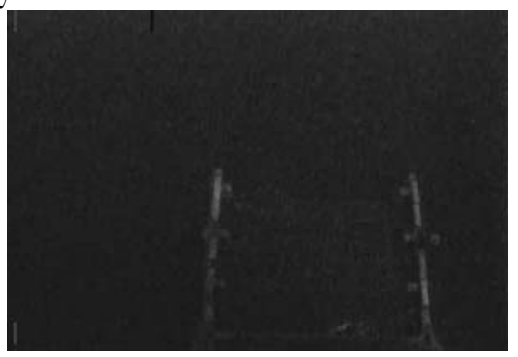

(b) Night

Fig. 2. The view of construction site: (a) Day and (b) Night 


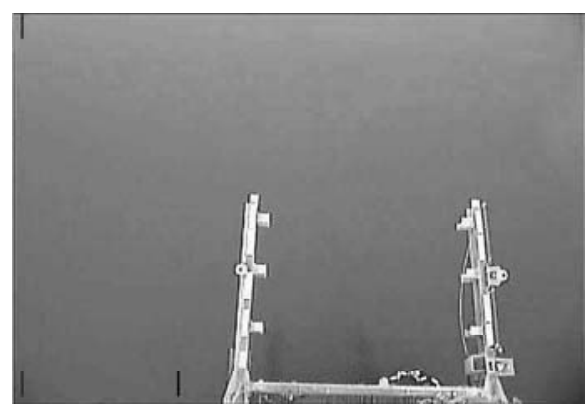

(a) Gray scale image

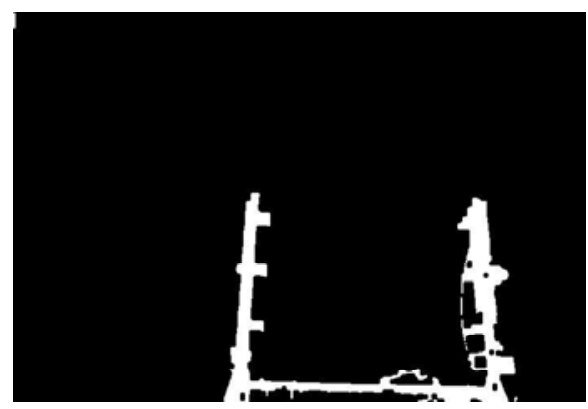

(c) Canny edge binary image with holles filled

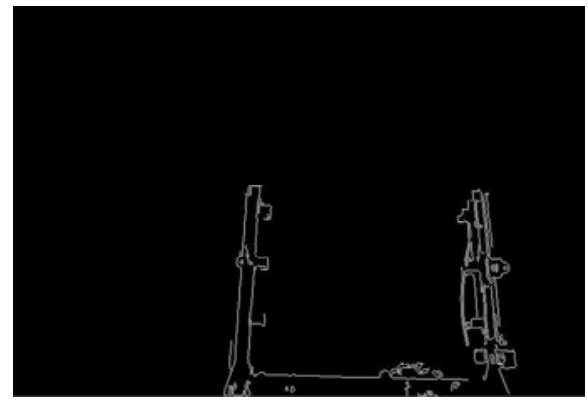

(b) Canny edge binary image

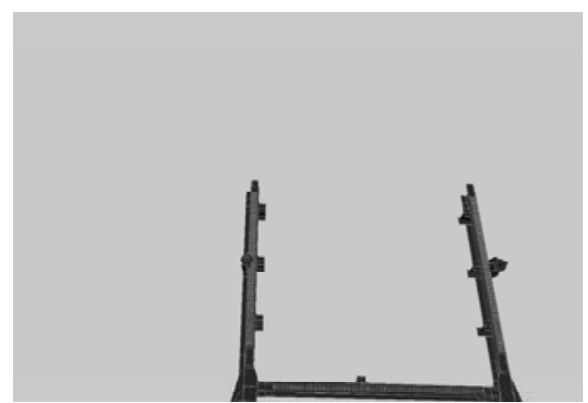

(d) 3D model

Fig. 3. The images of processed data and 3D model

\section{Object Segmentation}

A total of 14 structural components comprise one segment of the main span: two plate girders, three floor beams, three stringers, and six concrete panels. These 14 structural components are sequentially moved and installed by a derrick crane. Fig. 2 shows the day and night view of the process of two plate girders installation.

The acquired image data of the two plate girders were processed and analysed on a Matlab ${ }^{\mathrm{TM}}$ platform. Fig. 3 presents the processed images and the 3D CAD model of structural components. To perform the edge detection of the object of interest, the original RGB (Red, Green, and Blue) color image (Fig. 2 (a)) was converted to a gray scale image. The resultant image of the conversion process is presented at Fig. 3 (a). Next, the edge of the object of interest was detected by the canny edge detection algorithm using a $5 \times 5$ Gaussian filter with automatically selected optimum high threshold value $T_{H}$ and low threshold value $T_{L}$. The canny edge binary image of the two plate girder is shown in Fig. 3 (b). The gaps and holes of the detected edge were closed and filled with binary morphological closing and filling functions. The result of these two functions is presented at Fig. 3 (c). To compare the actual progress to the original plan, the 3D CAD model of the cable stayed bridge was generated in the Autodesk Revit Architecture Suite environment. By matching the processed image data (Fig. 3 (c)) with the 3D model (Fig. 3 (d)), the progress of the construction process can be measured.

\section{Conclusions}

This research presents the whole process of monitoring a cable stayed bridge construction, from the automated data acquisition to the data analysis. With the use of a CCTV camera and WLAN, the image data of the construction process were automatically transferred from the construction site to the head office storage device. To analyse the construction progress, image processing techniques along with the 3D CAD model were used for the comparison between the acquired data and as-planned data.

This preliminary research shows the possibility of automated process monitoring for civil structures such as cable stayed bridges using CCTV cameras and image processing techniques. Future study will focus on how to extract useful management information from the processed image data. This methodology is 
expected to lead to a fully automatic bridge construction progress monitoring system for effective construction management.

\section{Acknowledgement}

This work was supported by a Grant (KRF-2008-331-D00611) from the Korea Research Foundation and a Grant (07UrbanRenessanceB03) from High-Tech Urban Development Program funded by the Korean Ministry of Land, Transport, and Maritime Affairs.

\section{References}

[1] Abdel-Qader, I, Abudayyeh, Osama, and Kelly, M. E. (2003). "Analysis of Edge-Detection Techniques for Crack Identification in Bridge.” Journal of Computing in Civil Engineering, 17(4), 255-263.

[2] Bosche, F., and Haas, C. T. (2008). "Automated retrieval of 3D CAD model objects in construction range images." Automation in Construction, 17(4), 499-512.

[3] Brilakis, I. K., and Soibelman, 1. (2008). "Shape-Based Retrieval of Construction Site Photographs." Journal of Computing in Civil Engineering, 22(1), 14-20.

[4] Kim, H., Haas, C. T., Rauch, A. F., and Browne, C. (2002). "Dimensional Ratios for Stone Aggregates from 3D Laser Scans.” Journal of Computing in Civil Engineering, 16(3), 175-183.

[5] Kim, H., Haas, C. T., Rauch, A. F., and Browne, C. (2003). "3D Image Segmentation of Aggregates from Laser Profiling." Computer-Aided Civil and Infrastructure Engineering, 18(4), 254-263.

[6] Kim, H., Haas, C. T., and Rauch, A. F. (2004). "Automated Quality Assessment of Stone Aggregates Based on Laser Imaging and a Neural Network.” Journal of Computing in Civil Engineering, 18(1), 5864.

[7] Quiñones-Rozo, C. A., Hashash, Y. M. A., and Liu L. Y. (2008). "Digital Image Reasoning for Tracking Excavation Activities." Automation in Construction, 17(5), 608-622.

[8] Zou, J., and Kim, H. (2007). "Using HSV Color Space for Construction Equipment Idle Time Analysis." Journal of Computing in Civil Engineering, 21(4), 238-246. 